\title{
A comparison of self-reports and electrodermal activity as indicators of mathematics state anxiety. An application of the control-value theory
}

\author{
Anselm R. Strohmaier1, Anja Schiepe-Tiska2, \& Kristina M. Reiss1,2 \\ ıHeinz Nixdorf Chair of Mathematics Education, TUM School of Education, \\ Technical University of Munich, Munich, Germany \\ ${ }_{2}$ Centre for International Student Assessment, \\ Technical University of Munich, Munich, Germany
}

Article received 11 November 2018 / Article revised 13 December 2019 / Accepted 24 January 2020 / Available online 19 February

\begin{abstract}
In the present study with 86 undergraduate students, we related trait Mathematics Anxiety (MA) with two indicators of state anxiety: self-reported state anxiety and electrodermal activity (EDA). Extending existing research, we included appraisals of control and perceived value in hierarchical multiple regression analyses in accordance with the control-value theory of achievement emotions (Pekrun, 2006). Results showed that trait MA predicted self-reported state anxiety, while no additional variance was explained by including control and value. In contrast, we found no significant relation between trait MA and physiological state anxiety, but a significant, negative three-way interaction effect with control and value. Regression coefficients indicated that trait MA predicted physiological state anxiety, but only in the presence of negative perceived control and positive perceived value. Thus, our results support the control-value theory for physiological state anxiety, but not for self-reports. They emphasize the need to distinguish between trait and state MA, the advantages of adopting the control-value theory, and the benefits of using EDA recording as a supplemental assessment method for state anxiety.
\end{abstract}

Keywords: mathematics anxiety, electrodermal activity, galvanic skin response, control-value theory, state anxiety. 


\section{Introduction}

Mathematics Anxiety (MA) has a substantial impact on many students' academic and personal lives. It influences achievement in mathematics tests and classes (Hembree, 1990; Ma, 1999; Namkung, Peng, \& Lin, 2019). Moreover, students with high MA avoid mathematics in everyday life as well as in career and academic choices (Dowker, Sarkar, \& Looi, 2016; Ma, 1999). MA is common across countries, cultures, and ages (Dowker et al., 2016; Lee, 2009). In the 2012 study of the Programme for International Student Assessment (PISA), 30\% of students reported that they felt helpless when doing a mathematic problem (OECD, 2013b). At the same time, MA is a problem of increasing relevance. On average across OECD countries, MA increased significantly from PISA 2003 to PISA 2012 (OECD, 2013b). Thus, for educational research, it is important to understand how MA affects students when doing mathematics. Research has elaborated the distinction between (momentary) state anxiety (MAstate) and (habitual) trait Mathematics Anxiety (MAtrait), assessed through separate self-reports, but the findings left their relationship ambiguous (Goetz, Bieg, Lüdtke, Pekrun, \& Hall, 2013). Hence, merely assessing MAtrait cannot exhaustively explain how MA affects mathematical activities momentarily. Then again, directly assessing MAstate provides a challenge, because selfreports of state emotions might be unreliable (Pekrun \& Bühner, 2014). Among other physiological measures, electrodermal activity (EDA; also referred to as galvanic skin response; GSR) had sporadically been used as an indicator for MAstate in the 1980s, but its relationship with self-reports of MAstate or to MAtrait remained unclear. In this paper, we addressed this research gap by combining two novel approaches. First, we included and compared both self-reports and EDA as measures of MAstate. Second, we used the control-value theory of achievement emotions (Pekrun, 2006) as a framework to test their relation to MAtrait. Accordingly, we included appraisals of control and perceived value as moderators of the relation between MAtrait and MAstate.

\subsection{Mathematics Anxiety}

MA "involves feelings of tension and anxiety that interfere with the manipulation of numbers and the solving of mathematical problems in a wide variety of ordinary life and academic situations" (Richardson \& Suinn, 1972, p. 551). MA has an adverse effect on cognitive resources, independent of actual abilities (Ashcraft, 2007; Ashcraft \& Kirk, 2001; Maloney et al., 2013). Ashcraft and Kirk (2001) found that in a mental addition task, undergraduates with high MA showed a smaller working memory capacity that led to an increase in reaction time and errors. This first finding started an intensive line of research, largely confirming direct effects of MA on performance (for overviews, see Dowker et al., 2016; Suárez-Pellicioni, Núñez-Peña, \& Colomé, 2016). This influence is not limited to working memory capacity. For example, Maloney, Ansari, and Fugelsang (2011) found that high MA students suffer from low-level numerical deficits, like a less precise representation of numerical magnitude. Although most studies refer to MA as a unidimensional construct, a number of studies reported evidence that it consists of more than one factor, most prominently a cognitive component ("worry") and an affective component ("emotionality"; e.g., Ho et al., 2000; Lukowski et al., 2016; Wigfield \& Meece, 1988). These studies typically analyzed the factorial structure of questionnaires and related the dimensions to cognitive outcomes like mathematical achievement (e.g., Ashcraft \& Ridley, 2005; Lukowski et al., 2016).

\subsection{Trait and state Mathematics Anxiety}

While there are a large number of studies on MA, very few of them differentiate between MAstate and MAtrait (Goetz, Bieg, Lüdtke, Pekrun, \& Hall, 2013; Goldin, 2014). However, this distinction arguably is important when focusing on the effects of MA during mathematical activities. Self-reports of MAtrait refer to multiple, generalized mathematical situations (Bieg, Goetz, Wolter, \& Hall, 2015). In contrast, MAstate refers to the specific, current situation. Therefore, reports of MAtrait might be a good predictor for long-term effects of MA on learning or career and course choices (Dowker et al., 2016) but do not necessarily accurately predict MAstate during specific mathematical activities like tests or classes. When investigating the effects of MA during such activities, directly addressing MAstate seems to be more appropriate. 
Studies investigating the role of emotions in mathematics and of MA in particular predominantly focus on trait emotions rather than state emotions (Goetz et al, 2013; Goldin, 2014). Accordingly, an extensive number of findings have been gathered on effects, individual differences, and precursors of MAtrait (Dowker et al., 2016). In contrast, there are fewer studies on MAstate, often using qualitative analyses (Goldin, 2014). Yet, specific mechanisms explaining the impact of MAstate have rarely been reported for mathematics (Dowker, 2016).

The relationship between MAstate and MAtrait is ambiguous. On the one hand, a number of studies indicate a strong positive relation. For high MAtrait students, MAstate is considered a key explanation for a lower working memory capacity (Ashcraft \& Moore, 2009; Beilock, 2008). In his meta-analysis, Hembree (1990) reports a mean correlation of $r=.42$ between MAtrait and state anxiety. However, state anxiety was not necessarily assessed during specific mathematical activities in the four reported studies (e.g. Plake \& Parker, 1982). On the other hand, some studies indicate that there is a notable discrepancy between MAtrait and MAstate. Goetz et al. (2013) found that girls systematically report higher levels of MAtrait, but that this difference is not present in reports of MA state during mathematics tests or classes. This difference between reports of MAtrait and MAstate is largely explained by individual beliefs and perceptions of competence (Bieg et al., 2015; Goetz et al., 2013). Another reason for differences between MAtrait and MAstate might be that MA negatively affects achievement in mathematics through long-term avoidance behavior, but not during mathematical activities per se (Dowker et al., 2016): To avoid aversive consequences, MA state can even enhance motivation momentarily and lead to an increase in effort and strategy use during mathematics tests (Eysenck \& Calvo, 1992; Eysenck, Derakshan, Santos, \& Calvo, 2007). This indicates that MAtrait does not necessarily induce MAstate.

In general, state anxiety can have various cognitive and motivational-affective effects on learning and performance. Zeidner (2014) lists 15 specific deficits in information processing during learning caused by anxiety, which are likely to be transferable to MAstate. This includes cognitive deficits in areas like information encoding, information storage and processing, and information retrieval and production. Moreover, state anxiety is associated with physiological reactions. However, this has not been described for MAstate in particular, but only for state anxiety in general. Per definition, state anxiety is a "transitory emotional state consisting of feelings of apprehension, nervousness, and physiological sequelae such as an increased heart rate or respiration" (Wiedemann, 2015, p. 808). Among other aspects, state anxiety is thus characterized by increased arousal and activation of the autonomic nervous system (Steimer, 2002; Wiedemann, 2015). Accordingly, state anxiety does not only cause cognitive deficits, but also a physiological reaction.

In sum, existing studies mostly focus on MAtrait, while its relation to MAstate is left ambiguous. Thus, to better understand how MA affects learning not only over a longer period of time but also momentarily, additional research is needed. This refers both to the question of the relation between MAtrait and MAstate, as well as to the mechanisms and precursors of MAstate in particular. In the following, we propose a theoretical framework for investigating these questions.

\subsection{The control-value theory}

The control-value theory of achievement emotions (Pekrun, 2006) characterizes predictors of achievement emotions, including state anxiety. It states that appraisals of control and the perceived subjective value of an achievement situation are the most proximal predictors of achievement emotions. A low appraisal of control and a simultaneous high perceived value of the task are key determinants of state anxiety. In contrast, trait emotions, environmental factors, or former achievement are considered distal factors and are assumed to have a mostly indirect effect on state emotions. According to the control-value theory, MAtrait should therefore predict MAstate mostly indirectly, in association with low appraisals of control and a high subjective value. Several empirical studies support aspects of the control-value theory in mathematics (e.g., Niculescu, Tempelaar, Dailey-Hebert, Segers, \& Gijselaers, 2015). Frenzel, Pekrun, and Goetz (2007) found that MAtrait is associated with a pattern of low competence beliefs paired with high achievement values in mathematics. Extending the scope, research about attitudes and beliefs about competence in mathematics offers plenty of evidence supporting the control-value theory for other mathematical achievement emotions (for an overview, see Goldin et al., 2016). However, to our knowledge, no study implemented both MAtrait and MAstate as well as appraisals of control and perceived value in one model. 


\subsection{Assessing mathematics state anxiety}

To assess MAstate, research has mostly focused on qualitative research (see Goldin, 2014, for an overview). These approaches included retrospective interviews and videotaping, but the reliability of these methods has been questioned (Goldin, 2014). Using a more quantitative approach, Goetz et al. (2013) proposed short self-reports that could be used both for measuring anxiety during tests as well as during classes. The advantage of self-reports is that they can be used conveniently for experience-sampling and might be more reliable than observations. However, self-reports about achievement emotions might disrupt the current activity (Goldin, 2014). Moreover, it is questionable if self-reports can reflect an accurate evaluation of current emotions. In general, self-reports can only cover aspects of emotions that a person is aware of, depend on the use of language, and are subject to systematic biases, e.g. social desirability (Pekrun \& Bühner, 2014).

Consequently, other researchers have attempted to use physiological measures to directly investigate MA in performance situations (Dowker et al., 2016; Hannula, 2016), predominantly using neuropsychological methods (e.g., Lyons \& Beilock, 2012; Pletzer, Kronbichler, Nuerk, \& Kerschbaum, 2015). These studies revealed that MA activates brain areas linked to fear processing, disgust and pain processing, but they did not distinguish between MAtrait and MAstate (Artemenko, Daroczy, Nuerk, 2015; Suárez-Pellicioni et al., 2016).

State anxiety in general is associated with arousal and stress and with physiological reactions due to the activation of the autonomic nervous system. This leads to an increased heart rate and respiration, among other physiological reactions (Steimer, 2002; Wiedemann, 2015). This also holds for state anxiety in the context of education (Zeidner, 2014). Therefore, these specific physiological reactions can be assumed to be an indicator for MAstate. Some studies have assessed heart rate or cortisol secretion to monitor stress levels during mathematical tests (Dew, Galassi, \& Galassi, 1984; Faust 1992, as cited in Ashcraft, 2002; MattarellaMicke, Mateo, Kozak, Foster, \& Beilock, 2011; Pletzer, Wood, Moeller, Nuerk, \& Kerschbaum, 2010; Sarkar, Dowker, \& Cohen Kadosh, 2014). These studies produced mixed results. Mattarella-Micke et al. (2011) showed that cortisol secretion can be associated with high performance (for low MA students) or with low performance (for high MA students), probably associated with a working memory overload. In contrast, Pletzer et al. (2010) did not find a correlation between cortisol secretion and reports of MA, but used self-reports of MAtrait, not MAstate. A relation between heart rate and state anxiety has been shown in various fields (e.g. Kantor, Endler, Heslegrave, \& Kocovski, 2001), but has rarely been used in mathematics. Faust (as cited in Ashcraft, 2002) reported changes in heart rate when a highly math-anxious group performed mathematics tests of increasing difficulty. In contrast, Dew, Galassi, and Galassi (1984) found no substantial relation between heart rate and MAtrait or MAstate.

In addition to heart rate, Dew, Galassi, and Galassi (1984) observed physiological arousal during a timed mathematics test assessing participants' EDA. EDA are fluctuations in skin conductance due to an increase in sweat gland activity. Since sweat gland activity is associated with the autonomic nervous system activity, EDA is an established method to assess physiological reactions to arousal, concerns, or stress (Boucsein, 2012; Naveteur \& Freixa i Baqué, 1987; Nikula, 1991). In his overview of the method, Boucsein (2012) extensively reviewed applications and correlates of various measures of EDA. He concludes that EDA "can be regarded as a valid indicator for the strength of - mostly negative - emotions, for observing the course of psychological stress, and for objectively determining coping efficacy" (p. 521). Therefore, EDA can indicate state anxiety by detecting associated physiological reactions (Boucsein, 2012).

EDA has recently been used to observe emotions during educational processes like self-regulated and multimedia learning (Dindar et al., 2019; Mudrick, Taub, Azevedo, Price, \& Lester, 2017) and reading (Meer, Breznitz, \& Katzir, 2016). Dew et al. (1984) used various measures of EDA and different scales to assess MAtrait and MAstate, but found no relation between EDA and MAstate, and only a small relation between EDA and MAstate for one of their measures of EDA. As a possible explanation, they acknowledge that the challenge of comparing cognitively experienced anxiety and physiologically experienced anxiety might need a larger sample than their 31 students. Moreover, their study design did not include a baseline measure, which is generally advisable for data quality (Boucsein, 2012) and could indicate if EDA is indeed influenced by a mathematical test context. Thus, while their theoretical assumptions seem well-founded, the authors argue that their data was not sufficient for a meaningful interpretation (Dew et al., 1984).

In conclusion, MAstate has been assessed through qualitative methods, self-reports, and physiological measures. Physiological reactions are a vital aspect of anxiety in general and arguably of MAstate in particular, 
but previous research has not provided clear results concerning the relation between self-reports and physiological measures of MAstate, or the relation between MAstate and MAtrait in general.

\subsection{The present research}

So far, we have discussed that the relation between MAstate and MAtrait is not yet fully understood. In performance situations, MAstate might be stronger related to processes influencing mathematical thinking, like a reduction of working memory capacity. Therefore, taking into account MAstate seems important when analyzing effects of MA, but it can be assessed in different ways. While self-reports of MAstate are easy to obtain, they might suffer from systematic biases. As an alternative, some studies used physiological measures of stress and arousal instead of self-reports to assess MAstate in mathematical performance situations. Yet, these studies did either not address both MAstate and MAtrait or, in the case of Dew et al. (1984), did not show clear results. Moreover, no study did yet include appraisals of control or value to describe the relation between MA state and MAtrait in accordance with the control-value theory. We consider this a considerable gap in research on MA. We assume that the approach by Dew and colleagues (1984) to use EDA as an indicator for MAstate is more promising today, because the possibilities to record and analyze EDA have greatly improved. Particularly, the innovations in EDA recording offer better possibilities in observing the association between EDA and MAtrait, since they allow to assess MAstate more reliable and in an authentic environment. At the same time, using the control-value theory offers a better theoretical framework for the correlation between MA state and individual antecedents. It has been supported by a number of studies using self-reports and other methods to assess state anxiety, but to our knowledge, the control-value theory has not yet been utilized to analyze precursors of EDA.

\subsection{Hypotheses}

In the present study, we investigated the relation of MAtrait with two indicators for MAstate, the physiological measure EDA and self-reported state anxiety. We assessed MAstate both in a baseline context (a relaxation exercise) and a mathematics test. First, we assumed that the mathematics test would lead to an increase in both measures (hypothesis 1) and thus indicate that anxiety is successfully induced by the mathematics test. Second, we assumed that there is a relation between self-reported MAstate and EDA (hypothesis 2). Moreover, we anticipated that our findings would replicate the direct association between selfreported MAstate and MAtrait (Goetz et al., 2013; hypothesis 3a). We expected to find a similar relation between EDA and MAtrait, since EDA should reveal physiological arousal, which in turn is an indicator of MAstate (hypothesis 3b). According to the control-value theory, appraisals of control and subjective value were included as predictors. We expected that this would confirm the relation between these appraisals and both measures of MAstate (hypotheses 4a and 4b). Finally, the relation between MAstate and MAtrait should be higher when students report low control and high perceived value. Thus, we expected a negative three-way interaction between MAtrait, appraisals of control, and perceived value, on both measures of MAstate, respectively (hypotheses 5a and b).

\section{Method}

\subsection{Sample and procedure}

95 undergraduate students participated in the study. They gave written informed consent before participation. The study was conducted according to the Ethical Principles of Psychologists and Code of Conduct of the American Psychological Association from 2017. An ethics approval was not required by institutional guidelines or national regulations, in line with the guidelines of the German Research Foundation. Due to technical difficulties, 5 participants had to be excluded from the sample. Additionally, we excluded 4 students because of deviations of more than $3 S D$ in one of the assessed measures. The remaining participants were 86 undergraduate students ( 53 female) from programs other than mathematics, ranging from engineering 
to nutritional science. Mathematics students were not recruited as participants to avoid a bias in their beliefs and attitudes towards mathematics, as well as in their mathematical skills. The mean age was 23.2 years $(S D=4.07)$. Participants were recruited on campus and were paid 15 EUR for participation. During recruitment and before the experiment any indication of a mathematical content of the study was avoided. The study was described as a study investigating EDA during various tasks.

The individual sessions of the experiment took place in an office at the university containing only two tables, two chairs, and a closed closet. At the beginning of the experiment, the experimenter made participants familiar with the wristband assessing EDA. She then put the device on the wrist of the participant's nondominant hand and fitted it comfortably. After recording had started, participants were presented a 5-minute relaxation exercise via headphones. The exercise facilitated relaxation through breathing exercises, accompanied by an audio track that included sounds from nature to help promote a relaxing environment for the participant.

When the participant removed the headphones after the exercise, the experimenter immediately presented the first questionnaire assessing state-anxiety. After the participant finished the questionnaire, a first mathematical test was presented. The participant was asked to read the instruction carefully and then wait for the signal to start. All participants had 10 minutes to solve the test and received a short notice after 8 minutes. After the test, the participant answered the second state-questionnaire. The procedure was repeated for a second mathematics test. At the end of the experiment, trait and demographic data were assessed.

\subsection{Mathematics tests}

Both mathematics tests consisted of six items. Eleven items were taken from a pool of released items from the PISA-Study (OECD, 2013a); one item was adopted from the Trends in International Mathematics and Science Study (TIMSS, International Association for the Evaluation of Educational Achievement [IEA], 2013). Since research suggests that anxiety might have a larger influence for cognitively demanding tasks (Ching, 2017; Faust, Ashcraft, \& Fleck, 1996), we composed both tests to be fairly difficult. The overall solution rate of $42 \%(S D=21 \%)$ suggests that the tests were appropriately demanding.

The items covered a broad range of mathematical problems, ranging from geometry to statistics. They were based on the concept of mathematical literacy and therefore covered mathematical competencies beyond mere factual knowledge. The tasks required knowledge that all students should have achieved by the end of their compulsory education. For an overall achievement score, we coded each item according to the coding instructions from PISA and TIMSS $(0=$ incorrect, 0.5 = partially correct, $1=$ correct; OECD, 2013a; IEA, 2013) and calculated a sum score for all 12 items.

\subsection{Study measures}

We assessed MAtrait using the ANXMAT-Scale developed for the PISA-studies (five items, e.g. "I feel helpless when doing a mathematics problem", $\alpha=.87$; OECD, 2005). Participants answered on a 4-point Likert scale from 1, strongly disagree to 4, strongly agree. We assessed self-reported MAstate twice during the experiment according to Goetz et al., 2013, asking if participants felt anxious in the previous situation (1, definitely not to 4, definitely). Appraisals of control and perceived values were assessed after both tests and were task-specific. For appraisals of control, we used two items accounting for the controllability and probability of outcomes (e.g. "I think my competence in this area is ...", $\alpha=.78$ ) on a 7-point Likert-scale (1, low to 9, high; Engeser \& Rheinberg, 2008; Pekrun \& Perry, 2014). Appraisals of perceived value were assessed with the four-item cognitive preferences-scale by Kehr, Von Rosenstiel, and Bles (1997) on a 7-point Likert-scale (e.g. "It is important to me to solve the exercises"; 1 , not at all to 9, very much; $\alpha=.85$ ).

For EDA data collection during the relaxation exercise and the tests, we used an Empatica E4 wristband. The wristband is worn like a watch and measures skin conductance with two stainless steel electrodes at the inner wrist. The exosomatic non-invasive sensor applies a very small, non-perceptible alternating current with a peak value of $100 \mu \mathrm{A}$ at $1 \mathrm{~V}$ with an $8 \mathrm{~Hz}$ frequency. The $4 \mathrm{~Hz}$ signal is recorded on an integrated flash memory. 


\subsection{EDA data analyses}

EDA signals consist of two components. The tonic signal is influenced by medium-term factors like room temperature or physiological characteristics of the individual. It provides a level of skin conductance that is rather stable within some seconds. Even though the tonic signal can be an indicator for stress or anxiety, the phasic component of the signal is suited better to compare EDA between individuals and is commonly used as an indicator for state anxiety (Boucsein, 2012). Phasic components of the EDA signal are usually called responses, since they reflect a short peak in the signal. Responses can be specific responses to a stimulation, for example a bursting balloon. However, there are phasic responses that are not associated to any specific external stimulation, hence nonspecific. The frequency of these nonspecific responses in skin conductance is associated with stress and anxiety and is one of the most common measures for EDA (Boucsein, 2012). The phasic and the tonic components of an EDA signal overlap and need to be decomposed for analyses.

Data processing was carried out using MATLAB (V9.2.0) and the MATLAB-based software Ledalab (V3.4.9). The software applies Continuous Decomposition Analysis to extract the phasic signal (Benedek \& Kaernbach, 2010). After the extraction, any peak in the phasic signal bigger than $.01 \mu \mathrm{S}$ is counted as a response (Boucsein, 2012). For both phases of the experiment (relaxation and test), the number of events is then summed up and divided by the duration of the phase in minutes. The result is the frequency of nonspecific skin conductance responses per minute (SCR.freq). SCR.freq served as the measure for physiological MAstate.

\subsection{Analyses}

For hypothesis 1, we conducted a repeated measures ANOVA to test for differences in state anxiety during the relaxation and the test. To assess the relation between the two measures of $\mathrm{MA}_{\text {state }}$ and their relation to MAtrait (hypothesis 2 and 3), we calculated the correlations controlling for gender, achievement, and the respective baseline measures (see Sect. 3.1). For hypotheses 4 and 5, we adopted a 5-step hierarchical multiple regression model for both measures of MAstate as outcome variables (self-reported and physiological MAstate). All predictors except gender were z-standardized before the analyses. In step 1, we included the control variables as predictors. In step 2, we additionally included MAtrait. In accordance with the control-value theory, step 3 included appraisals of control and subjective value. In step 4, we included the interaction term between control and subjective value. Finally, step 5 included the interaction terms between MAtrait and appraisals of control and subjective value, respectively. Additionally, we included the three-way interaction between MAtrait, control, and subjective value.

\section{Results}

\subsection{Control variables}

Gender differences exist between self-reports of MA (Dowker et al., 2016). Moreover, because of physiological differences in the sweat gland density and activity, women tend to display a weaker EDA reactivity than men (Boucsein, 2012). Accordingly, our results revealed significant gender differences, with females showing weaker EDA, $t(84)=2.93, p=.004$, reporting higher MAtrait, $t(84)=-2.38, p=.020$, and lower control, $t(84)=2.37, p=.020$. No significant gender differences were found regarding self-reports of MAstate, $t(84)=-0.49, p=.626$, and perceived value $t(84)=0.02, p=.984$. Because of this general influence of gender, we included gender as a control variable in all following analyses.

In addition, achievement is associated both with trait anxiety (Ma, 1999) and with physiological reaction (Mattarella-Micke et al., 2011). In our data, we similarly found a significant relation between the test score and reports of MAtrait, $r(86)=-.28, p=.008$, self-reports of MAstate, $r(86)=-.31, p=.004$, and control, 
$r(86)=.51, p=.000$, respectively, but no significant relation between the test score and EDA, $r(86)$ $=.15, p=.177$, and perceived value, $r(86)=.07, p=.518$, respectively. Since our analyses focused on the interplay of MAtrait and MAstate, irrespective of achievement, we also controlled for the test score in the following analyses. For both measures of MAstate (self-reports and EDA), we used the data from the relaxation exercise as respective baseline measures.

\subsection{Main analyses}

\subsubsection{Descriptive results}

Table 1 provides the means and standard deviations for MAtrait and appraisals of control and perceived value. Additionally, mean scores and standard deviations for both measures of MAstate during the relaxation exercise and the test are included. For both measures, MAstate was significantly higher during the test compared to the relaxation exercise, confirming hypothesis 1 . While physiological MAstate increased from 15.43 events per minute to 20.04 events per minute $\left(F(85)=10.53, p=.002, \eta_{2}=.10\right)$, self-reported anxiety increased from 1.37 to $1.62\left(F(85)=17.23, p<.001, \eta_{2}=.17\right)$.

Table 1

Descriptive statistics and differences between MAstate in relaxation exercise and tests

\begin{tabular}{lcccc}
\hline Measure & $M$ & $S D$ & $F$ & part. $\eta^{2}$ \\
\hline Predictors & & & & \\
$\quad$ MAtrait & 2.23 & 0.80 & & \\
$\quad$ Control & 5.08 & 1.57 & & \\
$\quad$ Value & 5.21 & 1.05 & & \\
Self-reported MAstate & & & & .10 \\
$\quad$ Relaxation & 1.37 & 0.57 & $F(85)=10.53^{* *}$ & \\
$\quad$ Test & 1.62 & 0.69 & & .17 \\
Physiological MAstate & & & & \\
$\quad$ Relaxation & 15.43 & 12.70 & $F(85)=17.23^{* * *}$ & \\
$\quad$ Test & 20.04 & 15.65 &
\end{tabular}

Note. The unit for physiological state Mathematics Anxiety is SCR.freq [1/min].

$* * p<.01 * * * p<.001$.

\subsubsection{Correlations}

Table 2 provides correlations between all measures. All correlations were controlled for gender and test score and for the respective MAstate baseline during the relaxation exercise. Contrary to hypothesis 2, no significant correlation was observed between the two measures of MAstate $(r=.06, p=.63)$. MAtrait showed a moderate and significant correlation with self-reported MAstate $(r=.34, p=.002)$, but not with physiological $\mathrm{MAstate}(r=.08, p=.48)$, which supports hypothesis $3 \mathrm{a}$, but not $3 \mathrm{~b}$.

Including appraisals of control and perceived values, MAtrait correlated moderately and significantly with control and value $(r=-.38, p<.001 ; r=.24, p=.029)$. A significant, moderate correlation emerged between appraisals of control and self-reported MAstate $(r=-.29, p=.008)$, but not physiological MAstate $(r=-.05, p=.65)$. In contrast, appraisals of the perceived value were significantly related to physiological MAstate $(r=.29, p=.007)$, but not to self-reported MAstate $(r=.16, p=.15)$. Appraisals of control and perceived value showed no significant relation $(r=-.01, p=.95)$. 
Table 2

Correlations between measures of anxiety and appraisals of control and perceived value

\begin{tabular}{llllll}
\hline Measure & 1. & 2. & 3. & 4. & 5. \\
\hline 1. Self-reported MAstate & & & & & \\
2. Physiological MAstate & .06 & & & & \\
3. MAtrait & $.34^{* *}$ & .08 & & \\
4. Control & $-.29^{* *}$ & -.05 & $-.38^{* * *}$ & & \\
5. Value & .16 & $.29^{* *}$ & $.24^{*}$ & -.01 & \\
\hline
\end{tabular}

Note. Correlations of the two measures of state Mathematics Anxiety are controlled for their respective baseline. All correlations are controlled for gender and test score. $n=86$.

$* p<.05 * * p<.01 * * * p<.001$.

\subsubsection{Hierarchical multiple regression}

Results of the hierarchical multiple regressions are reported in Table 3. It displays only the predictors added in each step. For the full hierarchical models, see Appendix A.1. For the two regressions, we used the two measures of MAstate as outcome measures respectively. Inclusion of the control variables explained 58\% of the variance in physiological MAstate during the test $(p<.001)$, and $24 \%$ of the variance in self-reported MAstate $(p<.001)$.

For self-reported MAstate, step 2 revealed a significant relation between MAtrait and self-reported MAstate $(\beta=0.32, p=.002)$ that explained additional $9 \%$ of the variance in self-reported MAstate $(p=.002)$. Step 3 did not confirm a relation between appraisals of control or perceived value and self-reported MAstate $(\beta=-0.20$, $p=.090 ; \beta=0.09, p=.37)$. Step 4 did not reveal an interaction effect of control $\times$ value $(\beta=-0.00, p=.98)$, and step 5 revealed no three-way interaction effect of MAtrait $\times$ control $\times$ value $(\beta=-0.15, p=.20)$. Similarly, the interaction effects MAtrait $\times$ control and MA $\times$ value were not significant $(\beta=-0.06, p=.59 ; \beta=-0.23$, $p=.053$ ). These findings do not support hypothesis $4 \mathrm{a}$ or $5 \mathrm{a}$. Overall, the predictors explained $39 \%$ of the variance in self-reported MAstate $(p<.001)$.

We conducted the same hierarchical multiple regression for physiological MAstate. Contrary to selfreported MAstate, step 2 did not reveal a significant relation with MAtrait $(\beta=0.06, p=.48)$. In step 3 , adding appraisals of control and perceived value increased the $R_{2}$ significantly by $4 \%(p=.033)$. In this step, perceived value had a significant positive relation with physiological MAstate $(\beta=0.19, p=.009)$, while no relation was found for control $(\beta=-0.04, p=.65)$. Again, step 4 did not reveal an interaction of the control and value on MAstate $(\beta=0.04, p=.65)$. Contrary to self-reported MAstate, step 5 revealed a negative three-way interaction effect of MAtrait $\times$ control $\times$ value $(\beta=-0.23, p=.008)$, while the interaction effects MAtrait $\times$ control and MAtrait $\times$ value were not significant $(\beta=-0.06, p=.44 ; \beta=-0.01, p=.96)$. These effects explained an additional $4 \%$ of the variance in physiological MAstate $(p=.041)$. The three-way interaction effect is displayed in Figure 1 (right). For comparison, Figure 1 (left) displays the non-significant interaction for self-reported MAstate. Because there was no significant direct relation between MAtrait and physiological MAstate, the slopes are less steep in Figure 1 (right) than for self-reported MAstate. However, it illustrates that the slope of MAtrait on physiological MAstate increases for students appraising low control and high perceived value at the same time. These results support hypothesis $5 \mathrm{~b}$, but not hypothesis $4 \mathrm{~b}$. Overall, the predictors explained $66 \%$ of the variance in physiological MA state $(p<.001)$. 
Table 3

Hierarchical multiple regression analyses for physiological MAstate and self-reported MAstate

\begin{tabular}{|c|c|c|c|c|}
\hline \multirow[b]{3}{*}{ Added Predictor } & \multicolumn{4}{|c|}{ MAstate measure } \\
\hline & \multicolumn{2}{|c|}{ Self-reported MAstate } & \multicolumn{2}{|c|}{ Physiological MAstate } \\
\hline & $\Delta R_{2}$ & $\beta$ & $\Delta R_{2}$ & $\beta$ \\
\hline $\begin{array}{l}\text { Step } 1 \\
\text { Control variablesa }\end{array}$ & $.24 * * *$ & & $.58^{* * *}$ & \\
\hline Step 2 & $.09 * *$ & & .00 & \\
\hline MAtrait & & $0.32 * *$ & & 0.06 \\
\hline Step 3 & .03 & & $.04 *$ & \\
\hline Control & & -0.20 & & -0.04 \\
\hline Value & & 0.09 & & $0.19 * *$ \\
\hline Step 4 & .00 & & .00 & \\
\hline Control $\times$ Value & & -0.00 & & 0.04 \\
\hline Step 5 & .04 & & $.04 *$ & \\
\hline MAtrait $\times$ Control & & -0.06 & & -0.06 \\
\hline MAtrait $\times$ Value & & -0.23 & & -0.01 \\
\hline MAtrait $\times($ Control $\times$ Value $)$ & & -0.15 & & $-0.23 * *$ \\
\hline Total $R_{2}$ & $.39 * * *$ & & $.66^{* * *}$ & \\
\hline$n$ & 86 & & 86 & \\
\hline
\end{tabular}

aControl variables included respective baseline state Mathematics Anxiety, gender, and test score. $* p<.05 * * p<.01 * * * p<.001$.
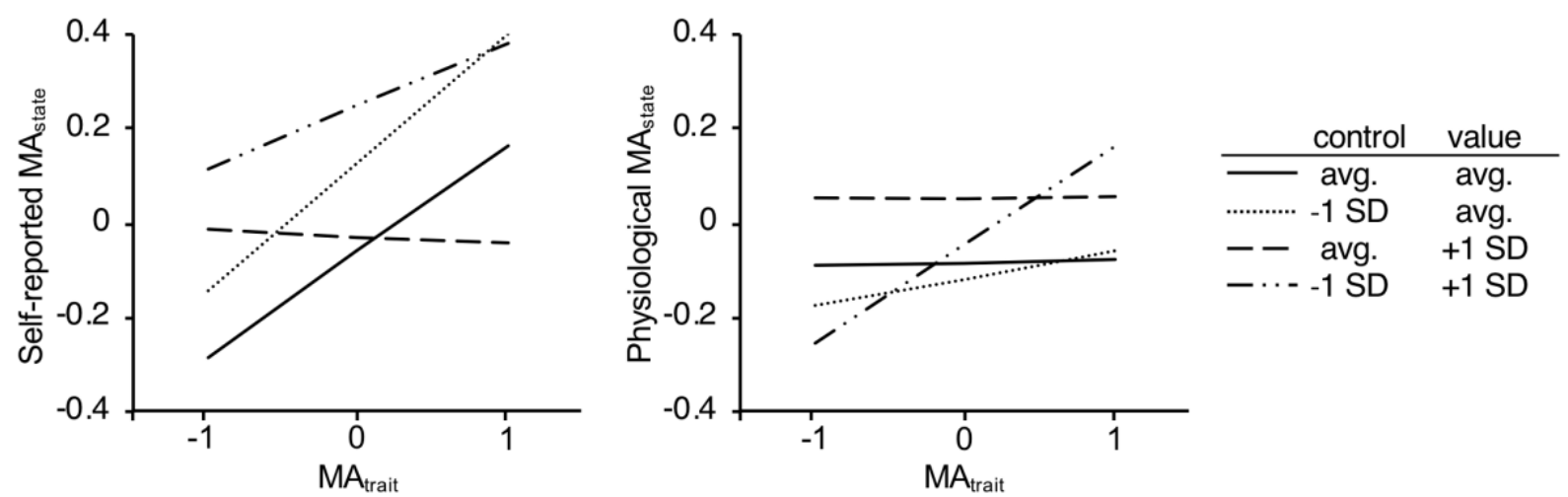

Figure 1. Relation between MAtrait and $\mathrm{MA}_{\text {state }}$ in dependence of control and perceived value.

\section{Discussion}

\subsection{Measures of state anxiety in mathematics tests}

In line with hypothesis 1 , we found significant differences between the relaxation exercise and the test for both measures of MAstate. This implies that the mathematics test induced anxiety compared to the relaxation exercise. However, descriptive analyses showed that self-reports of MAstate were relatively low in our study. 
This might be due to the fact that the experiment was a low-stakes test for the participants. We would assume that our result might emerge even stronger in a high-stakes test situation.

In contrast to our hypothesis, students' self-reports about MAstate and their physiological MA state were not significantly related. Our assumption had been that even though self-reports and physiological measures might differ to some extent, they should still refer to a similar MA state and hence be related. Judging from our results, the two measures might refer to conceptually different aspects of MAstate. Some researchers suggest that MAtrait is a multidimensional construct, usually differentiating between a cognitive and an affective dimension (Lukowski et al., 2016; Wigfield \& Meece, 1988). Similarly, physiological MAstate and self-reported MAstate as assessed in this study might refer to different facets of MAstate. Consequently, they might not necessarily be related. For example, EDA might be more associated with arousal and an affective, emotional dimension of MAstate. In contrast, self-reports might be more related to a cognitive dimension of MAstate that is associated with worries and cognitive resources (Ashcraft \& Moore, 2009; Beilock, 2008; Liebert \& Morris, 1967). Future research could include a multi-dimensional assessment of MA to address this possibility. Additionally, the measures might differ because of their differing mode of assessment (Pekrun \& Bühner, 2014). Self-reports might not be able to paint an adequate picture of achievement emotions, especially for a highly physiological emotion like anxiety (Pekrun \& Bühner, 2014). Furthermore, self-reports of MAstate might be subject to biases like social desirability (Pekrun \& Bühner, 2014) or stereotypes (Goetz et al., 2013).

\subsection{The relation between MAstate and MAtrait}

In line with Goetz et al. (2013), we found a significant relation between MAtrait and self-reported MAstate which was within the range of previous findings reported by Hembree (1990). Students with higher MAtrait also reported higher MAstate during a mathematical test. However, we did not find a relation between MAtrait and physiological MAstate. This finding is contrary to hypothesis $3 \mathrm{~b}$ but is in line with previous findings by Dew et al. (1984). Dew et al. (1984) proposed two explanations. First, the results might be viewed as questioning the construct validity of MAtrait scales. Since these scales have been further validated since then and worked as expected with regard to self-reported MAstate, this explanation seems unlikely. Alternatively, since students reporting MAstate need to evaluate their perceived anxiety cognitively, it is assumed that they might in part refer to generalized beliefs about mathematics. This might include the same resources as their evaluation of MAtrait (Bieg, Goetz, \& Lipnevich, 2014; Goetz et al., 2013), or students might even refer directly to their MAtrait when trying to evaluate MAstate. This would increase the relation between self-reported MAstate and MAtrait, but not between physiological MAstate and MAtrait.

\subsection{The control-value theory}

According to the control-value theory (Pekrun, 2006), MAstate should primarily be determined by appraisals of control and perceived value. These appraisals should also moderate the relation between MAtrait and MAstate. For the two measures of MAstate, the application of the control-value theory in the present study produced diverging results.

Both MAtrait and self-reported MAstate were related to appraisals of control. Nevertheless, the hierarchical multiple regression did not produce signs that appraisals of control or value play an important role for the relation between MAtrait and self-reported MAstate. Rather, this relation seemed to be direct. Hence, we did not find support for the control-value theory for self-reported MAstate. As was proposed above, the relation between MAtrait and self-reported MAstate might be increased by the similar mode of assessment. The resulting direct relation could overweight a possible indirect effect of appraisals of control and perceived value.

In contrast, physiological MA state showed a different pattern. In opposition to self-reported MA state, we did not find a direct correlation with MAtrait. However, we found strong support for the control-value theory in this second multiple regression analysis. First, perceived value was related to MA state, independent of MAtrait. Second, including appraisals of control and perceived value explained additional $8 \%$ of variance of physiological MAstate, which indicates a substantial contribution to its emergence. Third, the interplay between MAtrait, control, and value also was observed as expected. As illustrated in Figure 1 (right), high MAtrait was related to high MAstate, but only when students appraised their control low and their perceived value high. This effect is in line with the control-value theory, since MAtrait is considered a distal antecedent, whereas appraisals 
of control and perceived value are considered proximal causes of MAstate. These results further support the notion that the causal relation between MAtrait and the two measures of MAstate might be conceptually different.

\subsection{Limitations}

Using EDA comes with some immanent limitations, and only some of them can be overcome. For example, EDA is subject to physiological gender differences. This inhibits its practicality for inquiring the gender gap in MA. Even when controlling for a baseline value, differences in reactivity exist. In general, a large variance between students' EDA makes comparisons more difficult. In our study, we assessed the baseline value during a relatively short period of time. A more reliable value could be obtained through several hours or days of baseline recordings (Boucsein, 2012). Of course, such a study requires much more time. Lastly, even though MA is common in students of all ages, our specific sample cannot be overgeneralized. It needs to be verified if EDA recording can be useful in schools and for specific groups of students, for example high-anxiety students or younger students.

More generally, our test did not seem to induce a very strong emotional reaction. In order to generalize our findings to high-stakes testing which might cause more MAstate, additional studies are needed. Moreover, we followed Goetz at al. (2013) in using a single-item scale to assess self-reported MAstate. This keeps the disruption of the participants at a minimum but might result in some inaccuracies. Our results indicate that the scale was working properly, but future studies might try to assess MAstate at more occasions or check if the one-item scale is appropriately precise. Similarly, a number of different questionnaires exist to assess MA and general test anxiety. Comparing these questionnaires regarding their relation to EDA, particularly regarding cognitive and affective dimensions of these scales, could help to explain the absent relation between selfreported and physiological MAstate.

The relation between EDA and physiological arousal has been well established by previous research (Boucsein, 2012). However, other factors than MAstate might additionally influence EDA during mathematics tests. Future research could incorporate additional state measures that assess cognitive load or situational motivation to further narrow down the processes associated with EDA reactivity, and might support these findings through qualitative data like interviews or think-aloud-protocols. Until the validity of EDA as a measure of physiological MAstate is fully understood, results will always require a cautious discussion of limitations and different explanations.

The control-value theory is generalizable to various achievement emotions, including both trait and state emotions (Pekrun, 2006). In the current cross-sectional study, we focused on MAstate as an outcome, and task-specific appraisals of control and perceived value as moderators. Consequently, we considered MAtrait as a distal predictor. However, future studies could also consider MAtrait as an outcome itself. For analyzing effects of general appraisals of control and perceived value towards mathematics as predictors of MAtrait, longitudinal designs would be more advantageous.

\subsection{Conclusion}

Our study combined several innovative approaches that have emerged in research on MA within the last years. With the distinction between MAtrait and MAstate, we differentiated between two different facets of MA. Further, through the adoption of the control-value theory, we compared EDA recordings and common self-reports as a tool for observing MAstate and investigated their unique relations to MAtrait. Overall, we found that EDA was related to MAtrait, but that this relation only got visible when taking appraisals of control and perceived value into account. Students reporting high MAtrait were not necessarily more physiologically anxious during mathematical activities. Rather, a pattern of appraisals of low control and high perceived value accompanied that relation. Hence, with regard to EDA, our results were in line with the control-value theory, which on the other hand was not supported by self-reported measures of MAstate. In sum, our findings match the plea by Goetz at al. (2013) to consequently distinguish between MAtrait and MAstate in research on MA, as well as to additionally include physiological data in assessing emotions in learning. 
Furthermore, our results indicate that self-reports and physiological measures might refer to different aspects of MAstate. Thus, our results support theoretical considerations and empirical findings that self-reports of MAstate should be interpreted cautiously. Ultimately, we cannot decide if self-reports or EDA captured actual MAstate. Rather, the two measures both seem to be related to MAtrait, but in different ways. Therefore, we cannot conclude that EDA can make self-reports obsolete, but we propose that the assessment of EDA can provide additional information about underlying affective aspects of MAstate. Because of recent technical advances in recording and analyses of EDA, the method seems to offer a convenient addition to the common practice of self-reports. Furthermore, the advances in EDA-recording offer the possibility to conduct studies in the classroom during regular classes with hardly any disruption. We believe that our study can be a first step into this promising direction of in vivo research on MA.

As a next step, the relation to mathematical achievement should be investigated. In the recent study, we used a mathematics test, the goal of which was to trigger MA, but that was not designed to diagnose mathematical achievement in detail. Our preliminary results indicate that achievement might be differently associated with self-reported and physiological MAstate, but a study that assesses mathematical performance in more detail is needed to shed light on this question. Additionally, achievement under conditions of MAstate and no MAstate should be compared in a within-subject design, since EDA shows a notable variance between subjects. Similarly, using tests that are not mathematical could help to distinguish how specific MAstate is linked to mathematics. At the same time, using EDA for other domains or test anxiety in general, possibly using the control-value theory, might be an interesting and fruitful perspective for future research. Lastly, the relation to working memory capacity, which has proven to be a key factor in the effects of MA, should be taken into account. Ultimately, this knowledge could be used to design longitudinal and intervention studies that use EDA to observe the role of MAstate for learning processes or create ways to decrease MAstate in mathematics tests, possibly without necessarily tackling MAtrait.

With a number of questions remaining unanswered, our study is merely a first step in including EDA as an indicator for MAstate. Nevertheless, the results illustrate that self-reports only comprise one perspective on the multi-faceted phenomenon of Mathematics Anxiety, and that including EDA can be uniquely insightful.

\section{Keypoints}

We did not find a correlation between EDA and measures of state anxiety or trait Mathematics Anxiety, respectively.

Self-reported state anxiety correlated significantly with trait anxiety independent of appraisals of control and perceived value, which is in contrast to the control-value theory.

In line with the control-value theory, trait Mathematics Anxiety predicted physiological state anxiety when high perceived value and low control of the achievement situation were reported.

\section{Acknowledgments}

We would like to thank Ashley L. Johnson and Kathrin Ebenhöh for their contributions during data collection. This research was funded by the Federal Ministry of Education and Research (BMBF) and the Standing Conference of the Ministers of Education and Cultural Affairs of the Länder in the Federal Republic of Germany (KMK) [Grant number ZIB2016]. 


\section{References}

Artemenko, C., Daroczy, G., Nuerk, H.-C. (2015). Neural correlates of math anxiety - an overview and implications. Frontiers in Psychology 6, 1333. doi:10.3389/fpsyg.2015.01333

Ashcraft, M. H. (2002). Math Anxiety: Personal, Educational, and Cognitive Consequences. Current Directions in Psychological Science 11(5), 181-185. doi:10.111/1467-8721.00196

Ashcraft, M. H. (2007). Is math anxiety a mathematical learning disability? In D. B. Berch \& M. M. M. Mazzocco (Eds.), Why Is Math So Hard for Some Children? The Nature and Origins of Mathematical Learning Difficulties and Disabilities (pp. 329-348). Baltimore: Brookes.

Ashcraft, M. H., \& Kirk, E. P. (2001). The relationships among working memory, math anxiety, and performance. Journal of Experimental Psychology: General, 130(2), 224-237. doi:10.1037//00963445.130.2.224

Ashcraft, M. H., \& Moore, A. M. (2009). Mathematics anxiety and the affective drop in performance. Journal of Psychoeducational Assessment, 27(3), 197-205. doi:10.1177/0734282908330580

Ashcraft, M. H., \& Ridley, K. S. (2005). Math anxiety and its cognitive consequences: A tutorial review In J. I. D. Campbell (Ed.), Handbook of mathematical cognition (pp. 315-327). New York, NY: Psychology Press.

Beilock, S. L. (2008). Math performance in stressfull situations. Current Directions in Psychological Science, 17(5), 339-343. doi:10.1111/j.1467-8721.2008.00602.x

Benedek, M., \& Kaernbach, C. (2010). A continuous measure of phasic electrodermal activity. Journal of Neuroscience Methods, 190(1), 80-91. doi:10.1016/j.jneumeth.2010.04.028

Bieg, M., Goetz, T., \& Lipnevich, A. A. (2014). What students think they feel differs from what they really feel - academic self-concept moderates the discrepancy between students' trait and state emotional self-reports. PLOS ONE, 9(3). doi:10.1371/journal.pone.0092563

Bieg, M., Goetz, T., Wolter, I., \& Hall, N. C. (2015). Gender stereotype endorsment differentially predicts girls' and boys' trait-state discrepancy in math anxiety. Froniers in Psychology, 6(1404). doi:10.3389/fpsyg.2015.01404

Boucsein, W. (2012). Electrodermal Activity (2 ed.). New York: Springer.

Ching, B. H.-H. (2017). Mathematics anxiety and working memory: Longitudinal associations with mathematical performance in Chinese children. Contemporary Educational Psychology, 51, 99-113. doi:10.1016/j.cedpsych.2017.06.006

Dew, K. M. H., Galassi, J. P., \& Galassi, M. D. (1984). Math anxiety: relation with situational test anxiety, performance, physiological arousal, and math avoidance behaviour. Journal of Counseling Psychology, 31(4), 580-583. doi:10.1037/0022-0167.31.4.580

Dowker, A., Sarkar, A., \& Looi, C. Y. (2016). Mathematics anxiety: What have we learned in 60 years? Frontiers in Psychology, 7, 508. doi:10.3389/fpsyg.2016.00508

Dindar, M., Malmberg, J., Järvelä, S., Haataja, E., \& Kirschner, P. A. (2019). Matching self-reports with electrodermal activity data: Investigating temporal changes in self-regulated learning. Education and Information Technologies. doi:10.1007/s10639-019-10059-5

Engeser, S., \& Rheinberg, F. (2008). Flow, performance and moderators of challenge-skill balance. Motivation and Emotion, 32(3), 158-172. doi:10.1007/s11031-008-9102-4

Eysenck, M. W., \& Calvo, M. G. (1992). Anxiety and performance: the Processing Efficiency Theory. Cognition and Emotion, 6(6), 409. doi:10.1080/02699939208409696

Eysenck, M. W., Derakshan, N., Santos, R., \& Calvo, M. G. (2007). Anxiety and cognitive performance: Attentional Control Theory. Emotion, 7(2), 336-353. doi:10.1037/1528-3542.7.2.336

Faust, M. W., Ashcraft, M. H., \& Fleck, D. E. (1996). Mathematics anxiety effects in simple and complex addition. Mathematical Cognition, 2, 25-62. doi:10.1080/135467996387534

Frenzel, A. C., Pekrun, R., \& Goetz, T. (2007). Girls and mathematics - A „hopeless“ issue? A control-value approach to gender differences in emotions towards mathematics. European Journal of Psychology of Education, 22(4), 497-514. doi:10.1007/BF03173468

Goetz, T., Bieg, M., Lüdtke, O., Pekrun, R., \& Hall, N. C. (2013). Do girls really experience more anxiety in mathematics? Psychological Science, 24(10), 2079-2087. doi:10.1177/0956797613486989

Goldin, G. A. (2014). Perspectives on emotion in mathematical engagement, learning, and problem solving. In R. Pekrun \& L. Linnenbrink-Garcia (Eds.), International handbook of emotions in education (pp. 391-414). New York: Routledge. 
Goldin, G. A., Hannula, M. S., Heyd-Metzuyanim, E., Jansen, A., Kaasila, R., Lutovac, S., . . . Zhang, Q. (Eds.). (2016). Attitudes, beliefs, motivation and identity in Mathematics Education. An overview of the field and future directions. Hamburg: Springer Open.

Hannula, M. S. (2016). Introduction. In G. A. Goldin, M. S. Hannula, E. Heyd-Metzuyanim, A. Jansen, R. Kaasila, S. Lutovac, P. Di Martino, F. Morselli, J. A. Middleton, M. Pantziara, \& Q. Zhang (Eds.), Attitudes, beliefs, motivation and identity in Mathematics Education. An overview of the field and future directions (pp. 1-2). Hamburg: Springer Open.

Hembree, R. (1990). The nature, effects and relief of mathematics anxiety. Journal for Research in Mathematics Education, 21. doi:10.2307/749455

Ho, H.-Z., Senturk, D., Lam, A. G., Zimmer, J. M., Hong, S., Okamoto, Y., . . W Wang, C.-P. (2000). The affective and cognitive dimensions of math anxiety: A cross-national study. Journal for Research in Mathematics Education, 31(3), 362-379. doi:10.2307/749811

International Association for the Evaluation of Educational Achievement [IEA] (2013). TIMSS 2011 assessment. Chestnut Hill, MA: TIMSS \& PIRLS International Study Center.

Kantor, L., Endler, N. S., Heslegrave, R. J., Kocovski, N. L. (2001). Validating self-report measures of state and trait anxiety against a physiological measure. Current Psychology 20(3), 207-215. doi: 10.1007/s12144-001-1007-2

Kehr, H. M., Von Rosenstiel, L., \& Bles, P. (1997). Zielbindung, subjektive Fähigkeiten und intrinsische Motivation [Goal commitment, subjective abilities, and intrinsic motivation]. Paper presented at the 16th Colloquium of Motivational Psychology, Potsdam, Germany.

Lee, J. (2009). Universals and specifics of math self-concept, math self-efficacy, and math anxiety across 41 PISA 2003 participating countries. Learning and Individual Differences, 19(3), 355-365. doi:10.1016/j.lindif.2008.10.009

Liebert, R. M., \& Morris, L. W. (1967). Cognitive and emotional components of test anxiety: a distinction and some initial data. Psychological Reports, 20(3), 975-978. doi:10.2466/pr0.1967.20.3.975

Lukowski, S. L., DiTrapani, J., Jeon, M., Wang, Z., Schenker, V. J., Doran, M. M., . . Petrill, S. A. (2016). Multidimensionality in the measurement of math-specific anxiety and its relationship with mathematical performance. Learning and Individual Differences. doi:10.1016/j.lindif.2016.07.007

Lyons, I. M., \& Beilock, S. L. (2012). Mathematics Anxiety: Separating the math from the anxiety. Cerebral Cortex, 22, 2102-2110. doi:10.1093/cercor/bhr289

Ma, X. (1999). A meta-analysis of the relationship between anxiety toward mathematics and achievement in mathematics. Journal for Research in Mathematics Education, 30(5), 520-540. doi:10.2307/749772

Maloney, E. A., Ansari, D., \& Fugelsang, J. A. (2011). The effect of mathematics anxiety on the processing of numerical magnitude. Quarterly Journal of Experimental Psychology, 64(1), 10-16. doi:10.1080/17470218.2010.533278

Maloney, E. A., Schaeffer, M. W., \& Beilock, S. L. (2013). Mathematics anxiety and stereotype threat: shared mechanisms, negative consequences and promising interventions. Research in Mathematics Education, 15(2), 115-128. doi:10.1080/14794802.2013.797744

Mattarella-Micke, A., Mateo, J., Kozak, M. N., Foster, K., \& Beilock, S. L. (2011). Choke or thrive? The relation between salvary cortisol and math performance depends on individual differences in working memory and math-anxiety. Emotion, 11(4), 1000-1005. doi:10.1037/a0023224

Meer, Y., Breznitz, Z., \& Katzir, T. (2016). Calibration of self-reports of anxiety and physiological measures of anxiety while reading in adults with and without readig disability. Dyslexia, 22, 267-284. doi: $10.1002 /$ dys. 1532

Mudrick, N. V., Taub, M., Azevedo, R., Price, M. J., \& Lester, J. (2017). Can physiology indicate cognitive, affective, metacognitive, and motivational self-regulated learning processes during multimedia learning? Paper presented at the Annual Meeting of the American Educational Research Association (AERA), San Antonio, TX.

Namkung, J. M., Peng, P., \& Lin, X. (2019). The relation between Mathematics Anxiety and mathematics performance among school-aged students: A meta-analysis. Review of Educational Research, 89(3), 459-496. doi:10.3102/0034654319843494

Naveteur, J., \& Freixa i Baqué, E. (1987). Individual differences in Electrodermal Activity as a function of subjects' anxiety. Personality and Individual Differences, 8(5), 615-626. doi:10.1016/01918869(87)90059-6 
Niculescu, A. C., Tempelaar, D., Dailey-Hebert, A., Segers, M., Gijselaers, W. (2015). Exploring the antecedents of learning-related emotions and their relations with achievement outcomes. Frontline Learning Research 3(1), 1-17. doi:10.14786/flr.v3i1.136

Nikula, R. (1991). Psychological correlates of nonspecific skin conductance responses. Psychophysiology, 28(1), 86-90. doi:10.1111/j.1469-8986.1991.tb03392.x

$\mathrm{Ng}$, E. L., \& Lee, K. (2015). Effects of trait test anxiety and state anxiety on children's working memory task performance. Learning and Individual Differences, 40, 141-148. doi:10.1016/j.lindif.2015.04.007

OECD. (2005). PISA 2003 technical report. Retrieved from http://www.oecd.org/edu/school/programmeforinternationalstudentassessmentpisa/35188570.pdf

OECD. (2013a). PISA 2012 released mathematics items. Retrieved from https://www.oecd.org/pisa/pisaproducts/pisa2012-2006-rel-items-maths-ENG.pdf

OECD. (2013b). PISA 2012 results: ready to learn: students'engagement, drive and self-beliefs (Volume III): Pisa, OECD Publishing.

Pekrun, R. (2006). The control-value theory of achievement emotions: assumptions, corollaries, and implications for educational research and practice. Educational Psychology Review, 18(4), 315-341. doi:10.1007/s10648-006-9029-9

Pekrun, R., \& Bühner, M. (2014). Self-report masures of academic emotions. In R. Pekrun \& L. LinnenbrinkGarcia (Eds.), International handbook of emotions in education (pp. 561-579). New York: Routledge.

Pekrun, R., Lichtenfeld, S., Marsh, H. W., Murayama, K., \& Götz, T. (2017). Achievement emotions and academic performance: longitudinal models of reciprocal effects. Child Development, 88(5), 16531670. doi:10.1111/cdev.12704

Pekrun, R., \& Perry, R. P. (2014). Control-Value Theory of Achievement Emotions. In R. Pekrun \& L. Linnenbrink-Garcia (Eds.), International handbook of emotions in education (pp. 120-141). New York: Routledge.

Plake, B. S., \& Parker, C. S. (1982). The development and validation of a revised version of the Mathematics Anxiety Rating Scale. Educational and psychological measurement, 42(2), 551-557. doi:10.1177/001316448204200218

Pletzer, B., Wood, G., Moeller, K., Nuerk, H. C., \& Kerschbaum, H. H. (2010). Predictors of performance in a real-life statistics examination depend on the individual cortisol profile. Biological Psychology, 85, 410-416. doi:10.1016/j.biopsycho.2010.08.015

Pletzer, B., Kronbichler, M., Nuerk, H.-C., \& Kerschbaum, H. H. (2015). Mathematics anxiety reduces default mode network deactivation in response to numerical tasks. Frontiers in Human Neuroscience, 9, 202. doi:10.3389/fnhum.2015.00202

Richardson, F. C., \& Suinn, R. M. (1972). The Mathematics Anxiety Rating Scale: psychometric data. Journal of Counseling Psychology, 19(6), 551-554. doi:10.1037/h0033456

Sarkar, A., Dowker, A., \& Cohen Kadosh, R. (2014). Cognitive enhancement or cognitive cost: trait-specific outcomes of brain stimulation in the case of mathematics anxiety. The Journal of Neuroscience, 34 , 16605-16610. doi:10.1523/JNEUROSCI.3129-14.2014

Steimer, T. (2002). The biology of fear- and anxiety-related behaviors. Dialogues in Clinical Neuroscience, 4(3), 231-249.

Suárez-Pellicioni, M., Núñez-Peña, M. I., \& Colomé, À. (2016). Math anxiety: a review of its cognitive consequences, psychophysiological correlates, and brain bases. Cognitive, Affective, \& Behavioral Neuroscience, 16(1), 3-22. doi:10.3758/s13415-015-0370-7

Wiedemann, K. (2015). Anxiety and anxiety disorders. In J. D. Wright (Ed.), International Encyclopedia of the Social \& Behavioral Sciences (Second Edition) (pp. 804-810). Amsterdam: Elsevier.

Wigfield, A., \& Meece, J. L. (1988). Math anxiety in elementary and secondary school students. Journal of Educational Psychology, 80(2), 210-216. doi:10.1037/0022-0663.80.2.210

Zeidner, M. (2014). Anxiety in education. In R. Pekrun \& L. Linnenbrink-Garcia (Eds.), International handbook of emotions in education (pp. 120-141). New York: Routledge. 
Table A.1

Full hierarchical multiple regression analyses for physiological MAstate and self-reported MAstate

\begin{tabular}{|c|c|c|c|c|c|c|c|c|c|c|}
\hline \multirow[b]{3}{*}{ Predictor } & \multicolumn{10}{|c|}{ MAstate measure } \\
\hline & \multicolumn{5}{|c|}{ Self-reported MAstate } & \multicolumn{5}{|c|}{ Physiological MAstate } \\
\hline & $\Delta R_{2}$ & $B$ & $S E$ & $\beta$ & Sig. & $\Delta R_{2}$ & $B$ & $S E$ & $\beta$ & Sig. \\
\hline Step 1 & .24 & & & & .000 & .58 & & & & .000 \\
\hline Intercept & & 1.58 & 0.09 & & .000 & & 19.08 & 1.46 & & .000 \\
\hline Baseline & & 0.26 & 0.07 & 0.38 & .000 & & 11.43 & 1.19 & 0.73 & .000 \\
\hline Gender & & 0.10 & 0.14 & 0.07 & .417 & & 2.50 & 2.44 & 0.08 & .309 \\
\hline Test Score & & -0.22 & 0.07 & -0.32 & .002 & & 0.10 & 1.15 & 0.01 & .934 \\
\hline Step 2 & .09 & & & & .002 & .00 & & & & .480 \\
\hline Intercept & & 1.55 & 0.08 & & .000 & & 18.90 & 1.49 & & .000 \\
\hline Baseline & & 0.26 & 0.06 & 0.37 & .000 & & 11.23 & 1.23 & 0.72 & .000 \\
\hline Gender & & 0.19 & 0.14 & 0.14 & .161 & & 2.99 & 2.54 & 0.09 & .244 \\
\hline Test Score & & -0.16 & 0.07 & -0.24 & .016 & & 0.38 & 1.21 & 0.02 & .780 \\
\hline MAtrait & & 0.22 & 0.07 & 0.32 & .002 & & 0.88 & 1.24 & 0.06 & .480 \\
\hline Step 3 & .03 & & & & .178 & .04 & & & & .033 \\
\hline Intercept & & 1.54 & 0.08 & & .000 & & 18.92 & 1.45 & & .000 \\
\hline Baseline & & 0.26 & 0.06 & 0.37 & .000 & & 11.14 & 1.19 & 0.71 & .000 \\
\hline Gender & & 0.21 & 0.14 & 0.15 & .119 & & 2.91 & 2.48 & 0.09 & .245 \\
\hline Test Score & & -0.12 & 0.07 & -0.17 & .113 & & 0.20 & 1.29 & 0.01 & .881 \\
\hline MAtrait & & 0.16 & 0.07 & 0.23 & .036 & & -0.09 & 1.33 & -0.01 & .946 \\
\hline Control & & -0.14 & 0.08 & -0.20 & .090 & & -0.64 & 1.40 & -0.04 & .648 \\
\hline Value & & 0.06 & 0.07 & 0.09 & .365 & & 3.02 & 1.14 & 0.19 & .009 \\
\hline Step 4 & .00 & & & & .975 & .00 & & & & .649 \\
\hline Intercept & & 1.54 & 0.08 & & .000 & & 18.86 & 1.46 & & .000 \\
\hline Baseline & & 0.26 & 0.07 & 0.37 & .000 & & 11.11 & 1.20 & 0.71 & .000 \\
\hline Gender & & 0.21 & 0.14 & 0.12 & .125 & & 3.04 & 2.51 & 0.10 & .230 \\
\hline Test Score & & -0.12 & 0.07 & -0.17 & .122 & & 0.11 & 1.32 & 0.01 & .937 \\
\hline MAtrait & & 0.16 & 0.08 & 0.23 & .038 & & -0.11 & 1.34 & -0.01 & .934 \\
\hline Control & & -0.14 & 0.08 & -0.20 & .096 & & -0.53 & 1.43 & -0.03 & .712 \\
\hline Value & & 0.06 & 0.07 & 0.09 & .403 & & 3.20 & 1.21 & 0.20 & .010 \\
\hline Control $\times$ Value & & -0.00 & 0.06 & -0.00 & .975 & & 0.49 & 1.06 & 0.04 & .649 \\
\hline Step 5 & .04 & & & & .241 & .04 & & & & .041 \\
\hline Intercept & & 1.58 & 0.09 & & .000 & & 18.73 & 1.49 & & .000 \\
\hline Baseline & & 0.24 & 0.07 & 0.35 & .000 & & 11.21 & 1.18 & 0.72 & .000 \\
\hline Gender & & 0.18 & 0.14 & 0.12 & .210 & & 2.76 & 2.46 & 0.09 & .266 \\
\hline Test Score & & -0.10 & 0.08 & -0.14 & .203 & & -0.41 & 1.30 & -0.03 & .756 \\
\hline MAtrait & & 0.16 & 0.08 & 0.23 & .046 & & 0.10 & 1.33 & 0.01 & .942 \\
\hline Control & & -0.13 & 0.08 & -0.19 & .123 & & 0.52 & 1.43 & 0.03 & .087 \\
\hline Value & & 0.02 & 0.08 & 0.03 & .762 & & 2.16 & 1.25 & 0.14 & .716 \\
\hline Control $\times$ Value & & -0.06 & 0.07 & -0.10 & .413 & & 1.05 & 1.21 & 0.08 & .385 \\
\hline MAtrait $\times$ Control & & -0.03 & 0.06 & -0.06 & .586 & & -0.81 & 1.04 & -0.06 & .437 \\
\hline MAtrait $\times$ Value & & -0.17 & 0.08 & -0.23 & .053 & & -0.08 & 1.44 & -0.01 & .958 \\
\hline MAtrait $\times($ Control $\times$ Value $)$ & & -0.07 & 0.05 & -0.15 & .203 & & -2.44 & 0.90 & -0.23 & .008 \\
\hline Total $R_{2}$ & .39 & & & & .000 & & .66 & & & .000 \\
\hline$n$ & 86 & & & & & & 86 & & & \\
\hline
\end{tabular}

Note. Variables Baseline and Test Score were z-standardized before the regression. Gender is coded $0=$ female, $1=$ male. 